\title{
Phosphor bleibt spannend!
}

\author{
Arno Pfitzner*
}

\author{
Stichwörter: \\ Elementmodifikationen - Phosphor . \\ Strukturaufklärung
}

$D_{\text {i }}$ Suche nach neuen Elementmodifikationen ist heute im Wesentlichen von der Arbeit unter besonderen physikalischen Bedingungen geprägt, insbesondere hohen Drücken und oft auch hohen Temperaturen. Dies ist nicht verwunderlich, da die Entdeckung neuer Elemente und deren Charakterisierung bei Normalbedingungen doch eher ein Thema chemiehistorischer Literatur ist. Dennoch gibt es im Bereich der Hauptgruppenelemente immer wieder Überraschungen, wie die Entdeckung der Fullerene, für die im Jahr 1996 gar der Nobelpreis für Chemie an Curl, Kroto und Smalley verliehen wurde. Jedem Interessierten fällt neben Kohlenstoff sicher sofort ein weiteres Element ein, bei dem unter Berücksichtigung kinetischer Effekte mit neuen Modifikationen $\mathrm{zu}$ rechnen ist, dessen Zustandsdiagramm also noch weiße Flecken enthält: Phosphor. Da dieses Element schon seit etwa 350 Jahren bekannt ist, scheint dies zunächst verwunderlich, ist aber nachvollziehbar, wenn man sich vor Augen führt, wie vielfältig die Möglichkeiten zur gegenseitigen Verknüpfung von Phosphoratomen sind.

Böcker und Häser untersuchten 1995 systematisch mit theoretischen Methoden die energetischen Beziehungen zwischen den vielen denkbaren Verknüpfungsmustern des Phosphors. ${ }^{[1]}$ Diese Arbeit zeigte, dass es eine ganze Reihe von energetisch gleichwertigen Möglichkeiten gibt, z.B. Polymere aus

[*] Prof. Dr. A. Pfitzner

Institut für Anorganische Chemie

Universität Regensburg

93040 Regensburg (Deutschland)

Fax: (+49) 941-943-4983

E-mail: arno.pfitzner@chemie.uni-regensburg.de
Phosphor aufzubauen. Dies erklärt vermutlich auch die schwierige Aufklärung der Strukturen der verschiedenen Formen des so genannten roten Phosphors. Neben den vielatomigen Polymeren gibt es mit dem tetraedrischen $\mathrm{P}_{4}$ auch ein sehr kleines Phosphormolekül, das jedem Chemiker bekannt ist.

Die Entdeckung des Phosphors liegt schon lange zurück, aber erst jetzt scheint die Zeit endlich reif für eine ganze Reihe neuer experimenteller Befunde zu sein. So berichteten Ruck et al. ${ }^{[2]}$ über die Aufklärung der Kristallstruktur des so genannten faserförmigen Phosphors, der bereits 1969 von Thurn und Krebs postuliert worden war. ${ }^{[3]}$ Offenbar bedingt durch die Kristallisationsbedingungen werden die polymeren Phosphorröhren mit dem bekannten fünfeckigen Querschnitt entweder über Kreuz miteinander verknüpft (Hittorfscher oder violetter Phosphor) oder aber parallel, sodass Doppelröhren entstehen (faserförmiger Phosphor). Nach den jetzt vorliegenden Ergebnissen von Ruck et al. bildet sich Hittorfscher Phosphor ausgehend von amorphem rotem Phosphor sowohl aus Bleischmelzen als auch durch Erhitzen unter Zugabe von geringen Mengen Iod. Die faserförmige Modifikation wurde dagegen aus Blei nicht erhalten; sie entsteht kristallin in nachweisbaren Mengen am besten in einem Temperaturgradienten von ca. $10 \mathrm{~K}$ bei Temperaturen um $580^{\circ} \mathrm{C}$. Ruck et al. schließen aus ihren Beobachtungen, dass die Kristallisation der Röhren auf der Wand der Quarzglasampullen eine parallele Ausrichtung der Röhren unterstützt. Sie konnten die Kristallstruktur der faserförmigen Modifikation des Phosphors durch Röntgenbeugung an Einkristallen bestimmen (Abbildung 1) und durch hochaufgelöste Elektronenbeugungsaufnahmen die deutlichen Unterschiede zum Hittorfschen Phosphor aufzeigen.
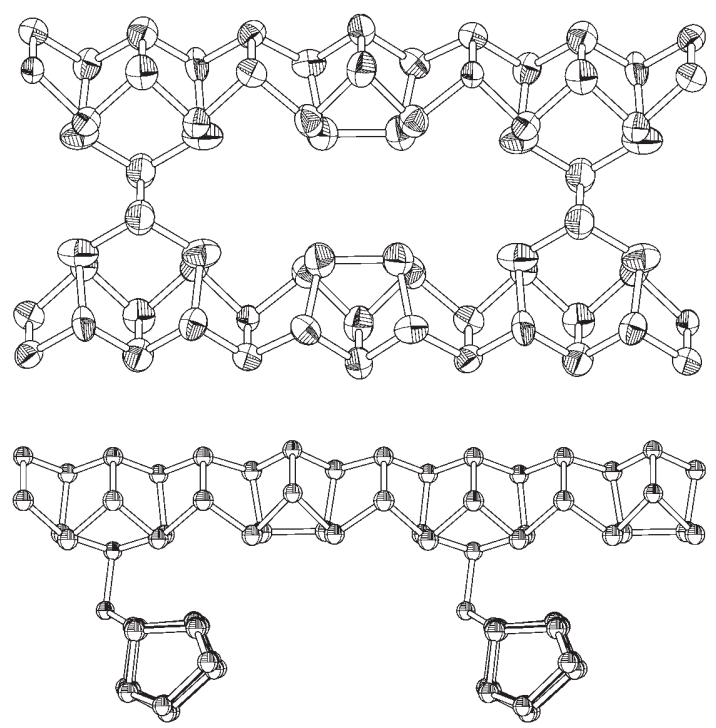

Abbildung 1. Verknüpfung der Phosphorstränge in faserförmigem Phosphor (oben; Daten aus Lit. [2]) und Hittorfschem Phosphor (unten; Daten aus Lit. [3]).

Umfangreiche theoretische Untersuchungen in Lit. [2] zeigten, dass die faserförmige Form anders als der Hittorfsche Phosphor keine $\pi$-Bindungsanteile für die P-P-Bindung zwischen den Strängen aufweist. Die faserförmige Modifikation hat an dieser Stelle eine trans-Konformation, während man die Verknüpfung im Hittorfschen Phosphor als gauche-Konformation betrachten kann. So lassen sich die an dieser Stelle 
signifikant unterschiedlichen Bindungslängen sehr gut erklären. Entgegen früheren Vermutungen ${ }^{[3]}$ ist die Dichte beider Modifikationen gleich. Daraus kann man schließen, dass in der Tat ausschließlich die Synthesebedingungen für das Entstehen der einen oder anderen Modifikation, und nicht etwa unterschiedliche thermodynamische Stabilitäten, entscheidend sind. Dies erklärt auch das gleichzeitige Entstehen von Hittorfschem und faserförmigem Phosphor aus der Gasphase. Rechnungen bestätigen die energetische Äquivalenz beider Allotrope.

Nach den theoretischen Untersuchungen von Häser ist davon auszugehen, dass neben diesen beiden unterschiedlich verknüpften Röhren aus Phosphor mit fünfeckigem Querschnitt auch die kürzlich aus ihren KupferiodidAddukten isolierten Polymere mit der translatorischen Einheit $\left[\mathrm{P}_{12}\right]^{[4]}$ (Abbildung 2) energetisch ähnlich günstig sind. Diese konnten jedoch bisher nicht in kristalliner Form erhalten werden, sondern fallen wegen der Synthesebedingungen und möglicherweise ihrer schlecht packbaren Struktur amorph in Form nanoskaliger Stäbchen an. Im Unterschied zu den kristallinen Modifikationen, die bei relativ hohen Temperaturen erhalten werden, lassen sich die nanoskaligen, nicht verknüpften Phosphorstäbchen bei Raumtemperatur isolieren.

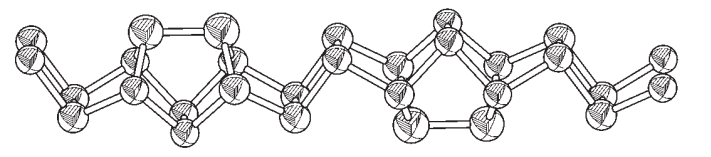

werden. ${ }^{[5]}$ Um diese Phase zu erhalten, wurde das Phasenumwandlungsverhalten von $\mathrm{P}_{4}$ bei schneller Temperaturänderung genutzt. Die Strukturlösung aus Pulverdaten erfolgte mithilfe moderner Methoden (simulated annealing und anschließende RietveldVerfeinerung). Eine genauere Analyse von $\gamma-\mathrm{P}_{4}$ durch theoretische Methoden zeigt, dass das freie Elektronenpaar an den Phosphoratomen die Kristallstruktur beeinflusst.

Damit ist innerhalb eines guten Jahres eine ganze Reihe von Alternativen zu Hittorfschem Phosphor synthetisiert und charakterisiert worden. Bei Betrachtung der Synthese- und Untersuchungsmethoden wird deutlich, dass nur durch die Kombination unterschiedlicher heute zugänglicher Methoden, wie hochauflösender Transmissionselektronenmikroskopie, FestkörperNMR-Spektroskopie und DFT-Rechnungen, eine zufrieden stellende Charakterisierung möglich war. Die Zeit ist also reif, die noch immer weißen Flecken im Zustandsdiagramm des Phosphors endlich zu füllen.

An dieser Stelle ist auch eine neue Arbeit über kristallinen, weißen Phosphor $\left(\mathrm{P}_{4}\right)$ zu erwähnen. Anfang des Jahres konnte durch Röntgenbeugung an pulverförmigen Proben die Kristallstruktur der $\gamma$-Phase von $\mathrm{P}_{4}$ bestimmt
Somit lassen sich die Abweichungen der Struktur von einer zu erwartenden "Idealstruktur" sehr gut durch eine Optimierung der Van-der-Waals-Kräfte zwischen den tetraederförmigen Molekülen erklären. ${ }^{[5]}$

Online veröffentlicht am 28. Dezember 2005

[1] S. Böcker, M. Häser, Z. Anorg. Allg. Chem. 1995, 621, 258.

[2] M. Ruck, D. Hoppe, B. Wahl, P. Simon, Y. Wang, G. Seifert, Angew. Chem. 2005, 117, 7788; Angew. Chem. Int. Ed. 2005, $44,7616$.

[3] H. Thurn, H. Krebs, Acta Crystallogr. Sect. B 1969, 25, 125.

[4] A. Pfitzner, M. F. Bräu, J. Zweck, G. Brunklaus, H. Eckert, Angew. Chem. 2004, 116, 4324; Angew. Chem. Int. Ed. 2004, 43, 4228.

[5] H. Okudera, R. E. Dinnebier, A. Simon, Z. Kristallogr. 2005, 220, 259. 